\title{
LARVAS FILOSOMAS DE LANGOSTA ESPINOSA Panulirus gracilis EN EL OCÉANO PACÍFICO COLOMBIANO, SEPTIEMBRE DE 2007
}

\author{
PHYLLOSOMA LARVAE OF THE SPINY LOBSTER Panulirus gracilis IN THE \\ COLOMBIAN PACIFIC OCEAN, SEPTEMBER, 2007
}

\author{
Juan Carlos Jaimes Martínez ${ }^{1}$ \\ Raúl Hernando López Peralta² \\ 1 y ${ }^{2}$ Grupo de Investigaciones en Hidrología Aplicada (Hidrobia), Programa de Biología. \\ Universidad Militar "Nueva Granada”. \\ Carrera 100 No. 101-80, Bogotá, D. C, Colombia. \\ juan.jaimes@unimilitar.edu.co ${ }^{1}$ y raul.lopez@unimilitar.edu.co ${ }^{2}$
}

Jaimes, J. y López, R. 2008. Larvas filosomas de langosta espinosa Panulirus gracilis en el océano Pacífico colombiano, septiembre de 2007. Boletín Científico CCCP, (15):33-45

\begin{abstract}
RESUMEN
En septiembre de 2007 se tomaron muestras de mesozooplancton en el océano Pacífico colombiano usando una red bongo con mallas de 294 y $520 \mu \mathrm{m}$. Se realizaron arrastres oblicuos hasta $184 \pm 42 \mathrm{~m}$ de profundidad en 23 estaciones. De las 46 muestras obtenidas se extrajeron las larvas filosoma para ser identificadas y cuantificadas y establecer su distribución. Se hicieron medidas biométricas de las larvas para presentar descripciones de los estadios de desarrollo. Sólo se capturaron nueve larvas de Panulirus gracilis de los estadios III, IV, V, VIII, IX y X, cuya densidad se estimó entre 4 y 14 ind $/ 1000 \mathrm{~m}^{3}$ por estación. Se evidenció una amplia distribución horizontal, observando larvas hasta 370 millas náuticas de la costa. Aunque la mayor captura se realizó en la noche y en cuarto creciente, no fue posible relacionar esto con la migración circadiana de las filosomas, por su bajo número y por el tipo de muestreo. La presencia de diferentes estadios de desarrollo en una misma estación sugirió una mezcla de diversos desoves. Todas las fases larvarias tuvieron menor tamaño que las de otras regiones, probablemente debido a factores biológicos y ambientales no considerados.
\end{abstract}

Palabras clave: Filosoma, Panulirus gracilis, langosta espinosa, meroplancton, Pacífico colombiano, corrientes, factores ambientales, factores biológicos.

\begin{abstract}
In September of 2007 mesozooplankton samples were taken from the Colombian Pacific Ocean using a bongo net, with meshes of 294 and $520 \mu \mathrm{m}$. Oblique hauls were carried out to a depth of $184 \pm 42 \mathrm{~m}$ in 23 stations. Of the 46 obtained samples the phyllosoma larvae were extracted to be identified and to be quantified establishing its distribution. Biometric measurements were made on larvae to present descriptions of the development stages. Only nine larvae of Panulirus gracilis of the stages III, IV, V, VIII, IX and X were caught, which density was estimated between 4 and 14 Ind $/ 1000 \mathrm{~m}^{3}$ per station. A wide horizontal distribution was demonstrated, observing larvae as futical miles offshore. Although the highest capture was realized at night and in first quarter, it was not possible to relate this with the circadian migration of phyllosoma, because of its low number and the type of sampling. The presence of different development stages in a same station suggested a mixing of different spawnings. All larval phases had smaller size than those of other regions, probably due to biological and environmental factors not considered.
\end{abstract}

Key words: Phyllosama, Panulirus gracilis, spiny lobster, meroplancton, Colombian, Pacific Ocean, current, environmental factors, biological factors. 


\section{INTRODUCCIÓN}

En el Pacífico Oriental se registran las langostas espinosas $P$. interruptus, $P$. pascuensis, $P$. penicillatus, $P$. inflatus y $P$. gracilis. La última en Baja California, México, (Lozano et al, 1982; Briones et al, 1997), Nicaragua (Velásquez y Gutiérrez, 2006), Costa Rica (Báez, 1983), Panamá (Guzmán et al, 2008), Ecuador (López y Barragán, 1974), las islas oceánicas Los Cocos (Panamá), Galápagos (Ecuador) (Holthuis, 1991; Lipcius y Cobb, 1994) y Paita (Perú) (Holthuis y Villalobos, 1962). En el océano Pacífico colombiano (OPC) aparece en las islas Malpelo y Gorgona, y en Bahía Málaga, junto con $P$. inflatus y $P$. pecillinatus (Abele, 1975; Lemaitre y Álvarez, 1992; Lazarus y Cantera, 2007).

$P$. gracillis presenta once estadios larvarios que se desarrollan como meroplancton en un tiempo estimado de nueve meses (Báez, 1983), al igual que la mayoría de los palinúridos. La ontogenia es bastante compleja y su desarrollo desde huevo hasta adulto se estima entre 2.5 y 3.5 años, tiempo en el cual pasa por varios hábitats. Las larvas eclosionan como filosomas planctónicas, se dispersan con las corrientes y recorren grandes distancias, siendo este mecanismo uno de los más importantes que inciden en la distribución de los palinúridos. Cumplida la metamorfosis de la última larva $(\mathrm{XI})$ al estadio puérulos, se acercan a la zona costera, se refugian en áreas bentónicas someras y en un corto período pasan a postpuérulos. Los juveniles y adultos habitan aguas más profundas y se desplazan en particular sobre sustratos rocosos o de cascajo-arena, tolerando aguas bastante turbias encontrándose hasta los 200 m de profundidad (Báez, 1983; López y Barragán, 1974; Briones et al, 1981; Cruz et al, 1990, 1995; Briones et al, 1997; Muñoz et al, 2000; Lazarus y Cantera, 2007).

En el Pacífico Oriental la mayor presión pesquera se da sobre $P$. inflatus y $P$. gracilis (Briones y Lozano, 1982, 1992; Martínez y Chávez, 1985; Holthuis, 1991; Guzmán et al, 2008). En OPC la pesca artesanal de estos palinúridos aporta únicamente el $2 \%$ de la extracción total de langostas en Colombia, porcentaje muy inferior al obtenido por $P$. argus en el Caribe colombiano, que aporta el 97\% a esta pesquería, por encima de $P$. laevicauda y $P$. guttatus (Inpa, 2001; ICA, 2006).

A pesar que la investigación sobre los palinúridos en Colombia se inició a finales de los años 60 (Alegría, 1971), desde entonces se ha enmarcado un especial enfoque hacia las pesquerías (Riveros, 1972; Arango y Márquez, 1995; Gallo et al, 2001; Borda y Cruz, 2006; Cruz et al, 2007), con énfasis en $P$. argus, resultando un gran vacío de información en cuanto a la dinámica de las fases larvales y postlarvales (Jaimes et al, 2004), con algunos trabajos no publicados (Martínez, 1970; Córdoba, 1997; Arango et al, 1999; Arango et al, 2001; Gareth, 2000; Jaimes, 2000; Medellín, 2005).

La escasa información sobre la dinámica planctónica de $P$. gracilis en el Pacífico colombiano, así como su relativa importancia en las pesquerías -de hecho sólo se presenta extracción artesanal-, enmarcan la relevancia de este documento que describe algunas etapas larvarias y estima su distribución y abundancia en septiembre de 2007. Este tipo de información revela algunas generalidades sobre los estadios larvarios de $P$. gracilis presentes en el OPC y motiva una mayor profundización en el tema.

\section{Área de Estudio}

El OPC se ubica entre los $01^{\circ} 30^{\prime \prime}$ y $07^{\circ} 10^{\prime \prime}$ de Latitud Norte y los $77^{\circ} 40^{\prime \prime}$ y $85^{\circ} 00^{\prime \prime}$ Longitud Oeste, con un área de $367823 \mathrm{~km}^{2}$ y unos $2188 \mathrm{~km}$ de línea costera, presentando un litoral rocoso que se distribuye en aproximadamente $636 \mathrm{~km}$ lineales localizados, en su mayoría, de Cabo Corrientes hacia la frontera con Panamá, en Bahía Málaga y en las islas Gorgona y Malpelo (Invemar, 2005). Hacia el norte, la plataforma continental, cuya distancia a la línea de costa no supera las cinco millas náuticas, presenta fondos lodosos terrígenos; hacia el sur se extiende hasta unas 30 millas náuticas, en cuyo fondo predomina un sustrato arenoso terrígeno. El OPC tiene como límites submarinos dos cordilleras que se unen en la plataforma de Galápagos, elevándose de 3700 a $1000 \mathrm{~m}$ de profundidad, lo que impide el intercambio de aguas profundas (Figura 1), (Dimar, 2000; CCCP, 2002).

Los desplazamientos latitudinales de la zona de convergencia intertropical y El Niño-Oscilación del Sur, moldean en gran medida la variabilidad de los vientos, precipitaciones, temperatura y salinidad superficiales en todo el OPC, lo cual afecta su hidrodinámica, que manifiesta surgencias todo el año, más extensas e intensas en la época menos lluviosa (marzo) (CCCP, 2002). Durante ésta se observa una mayor homogeneidad en la columna de agua, siendo más fría, con un termoclina más somero, mientras que en la época más lluviosa 
(agosto) hay mayor estratificación de las masas de agua y hacia la costa se presentan aguas cálidas y una termoclina más profunda (Milano y Bejarano, 1994; CCCP, 2002).

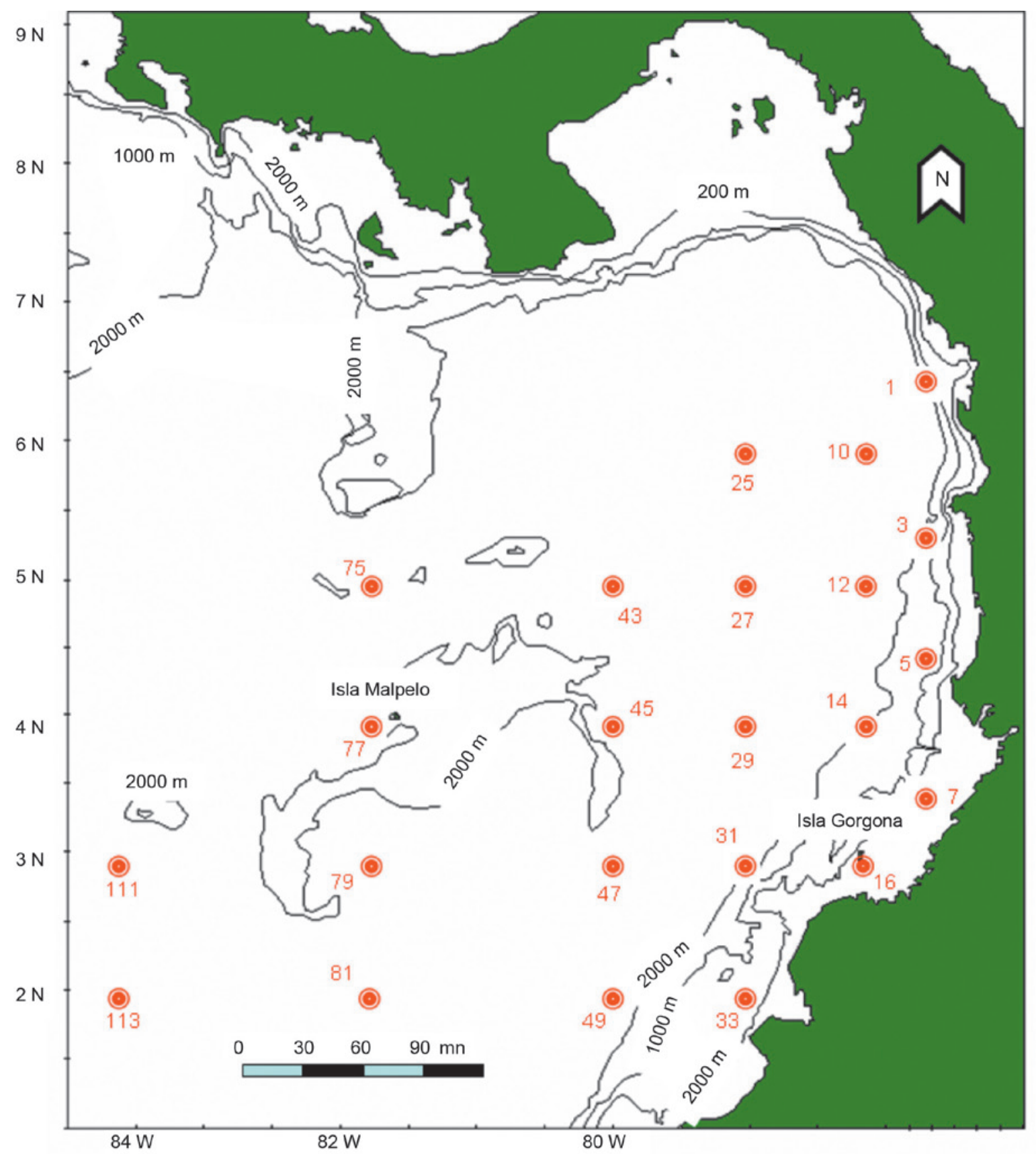

Figura 1. Ubicación de las 23 estaciones de muestreo de mesozooplancton en el OPC en septiembre de 2007 (tomado y modificado de Dimar, 2000).

\section{Metodología}

Los muestreos, realizados entre el 4 y el 28 de septiembre de 2007, se efectuaron en 23 estaciones de las 114 que conforman la malla de muestreo del Estudio Regional del Fenómeno El Niño (ERFEN)
(Figura 1) (CCCP, 2002). Se realizaron arrastres oblicuos hasta una profundidad de $184 \pm 42 \mathrm{~m}$, empleando una red bongo con apertura de $60 \mathrm{~cm}$, longitud de $1.85 \mathrm{~m}$ y mallas de 294 y $520 \mu \mathrm{m}$. Para determinar el volumen de agua filtrada se 
utilizó un flujómetro. Las muestras se fijaron con formaldehído al $10 \%$ en agua de mar, en el caso de la malla de $294 \mu \mathrm{m}$ (análisis morfométrico) y en alcohol al 99.9 \% la de $520 \mu \mathrm{m}$ (posterior análisis molecular).

El trabajo en el laboratorio incluyó la extracción de las filosomas de todas las muestras obtenidas por estación (i. e. 46 muestras). Se utilizó un estereoscopio y un microscopio con cámaras E4-Z10 y una cámara digital A620 de 7.1 MP, para la toma de imágenes que acompañan las descripciones. La selección de los datos biométricos, las identificaciones y las descripciones se soportaron en los trabajos de Báez (1983), Martínez y Chávez (1985), Dupré y Guisado (1996) y Rivera y Mujica (2004), teniendo en cuenta el tamaño y forma del cefalón, el desarrollo de las antenas, anténulas, maxilípedos, abdomen, telson y pleópodos, y el número de pereiópodos y espinas coxales y exopodales. Los especímenes se preservaron en formaldehído al $4 \%$ con $5 \mathrm{ml}$ de glicerina (Steedman, 1976).

Las abundancias se estandarizaron a $1000 \mathrm{~m}^{3}$, de acuerdo con Smith y Richardson (1979), así:

$$
\begin{aligned}
& \mathrm{N}=1000 \mathrm{~m}^{3 *} \mathrm{n} / \pi^{*} \mathrm{r}^{2 *} \mathrm{~d} \\
& \text { Donde, } \\
& \mathrm{N} \quad \text { Es el número de individuos } / 1000 \mathrm{~m}^{3} \\
& \mathrm{~N} \quad \text { Número de individuos en cada arras- } \\
& \text { tre } \\
& \pi * r^{2}{ }^{*} \mathrm{~d} \quad \text { Volumen de agua filtrada en cada } \\
& \text { arrastre } \\
& \mathrm{R} \quad \text { Radio de la red } \\
& \text { D l.cos }
\end{aligned}
$$

(La información sobre distribución y densidad se graficó en mapas elaborados con los programas Surfer 8 y AutoCAD 2007).

Los arrastres realizados de 6:00 am a 6:00 pm fueron considerados diurnos y de 6:01 pm a 5:59 am nocturnos. Las fases lunares durante el período de muestreo (04-28.Sep.07) fueron: cuarto menguante (04-08), luna nueva (09-15), cuarto creciente (1618) y luna llena (25-28).

\section{Resultados y Discusión}

\section{Composición, abundancia y distribución}

Se encontraron únicamente nueve filosomas de $P$. gracilis, en tan sólo cinco de las 23 estaciones (17\%). Según las características morfológicas descritas por Báez (1983) pertenecen a los estadios III, IV, V, VIII, IX y X. La falta de otros estadios puede obedecer a la dispersión que sufren las larvas de palinúridos y otras larvas de crustáceos a causa de las corrientes, como lo señalan Olvera y Ordóñez, (1988); Rivera y Mujica, (2004) y Lazarus y Cantera, (2007), lo que hace más aleatoria su captura.

A pesar del reporte de $P$. inflatus y $P$. pecillinatus en la costa del OPC, no se detectaron sus estadios larvarios, condición que puede relacionarse con las bajas densidades de adultos, como lo sugiere su nula participación en la pesca artesanal de langosta, soportada en su totalidad por $P$. gracilis en el área de estudio (Inpa, 2001). En relación con lo anterior, Cruz (1999) señala que la densidad de las poblaciones postlarvarias depende en gran medida de la dinámica reproductiva y la talla del stock reproductor; Baisre (1976), por su parte, menciona que algunas diferencias en las densidades larvarias de palinúridos pueden obedecer a discrepancias en los patrones de distribución. También se debe considerar que el mantenimiento de las poblaciones locales de adultos depende de la retención de larvas y su posterior acercamiento a las áreas de origen, de la migración de juveniles o adultos y la llegada de larvas provenientes de otras regiones (Scheltema, 1986; Briones y Gutiérrez 1991; Briones, 1994).

La densidad entre las cinco estaciones se estimó entre 4 y $14 \mathrm{lnd} / 1000 \mathrm{~m}^{3}$. La más alta se halló hacia el norte, en la estación intermedia 25 (14 Ind/1000 $\left.\mathrm{m}^{3}\right)$, seguida de la $45\left(9 \mathrm{lnd} / 1000 \mathrm{~m}^{3}\right)$, ubicada hacia el centro del área de estudio. Los valores intermedios $\left(7 \mathrm{lnd} / 1000 \mathrm{~m}^{3}\right)$ se registraron en las estaciones costera 1 y oceánica 111, al norte y suroccidente, respectivamente, y el valor más bajo (7 Ind./ $1000 \mathrm{~m}^{3}$ ) en la estación intermedia 27 (Tabla I, Figura 2).

En cuanto a las observaciones nictimerales y por fase lunar, seis de las nueve filosomas se capturaron en la noche y ocho entre luna nueva y cuarto creciente, $i$. e. durante la menor intensidad lumínica. En principio, estos datos podrían atribuirse a migraciones verticales, que exhiben las larvas de algunos palinúridos con una mayor abundancia cerca 
Tabla I. Densidad de las filosomas de $P$. gracilis capturadas en el OPC durante septiembre de 2007.

\begin{tabular}{|c|c|c|c|c|c|c|}
\hline Estación & $\begin{array}{c}\text { Distancia de la } \\
\text { costa (mn) }\end{array}$ & Fecha & Estadio & $\begin{array}{c}\text { Ind/ } \\
1000 \mathrm{~m}^{3}\end{array}$ & Fase lunar & Malla ( $\mu \mathrm{m})$ \\
\hline 1 & 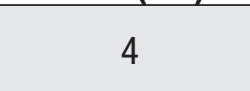 & $10 . \operatorname{Sep} .07^{*}$ & $\begin{array}{l}\text { III } \\
\text { IV }\end{array}$ & 7 & Luna nueva & 294 \\
\hline 25 & 100 & 18.Sep.07* & $\begin{array}{c}\text { X } \\
\text { IX } \\
\text { III }\end{array}$ & 14 & Cuarto creciente & 520 \\
\hline 27 & 93 & 17.Sep.07 & IX & 4 & & \\
\hline 45 & 153 & 13.Sep.07 & $\underset{\mathrm{V}}{\mathrm{V}}$ & 9 & Luna nueva & $\begin{array}{l}294 \\
520\end{array}$ \\
\hline 111 & 370 & 26.Sep.07 & IV & 7 & Luna Ilena & 294 \\
\hline
\end{tabular}

*Muestreo nocturno.

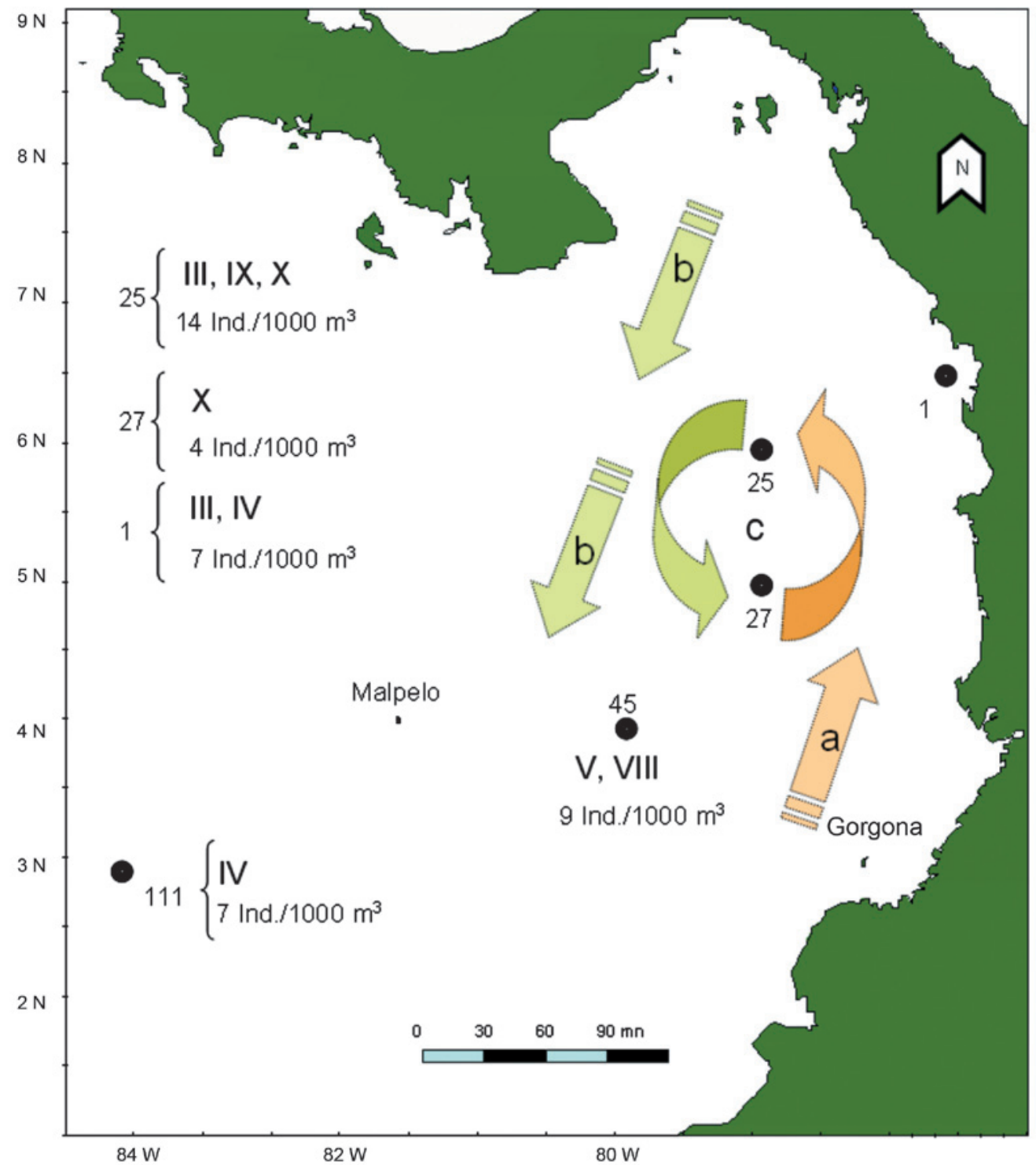

Figura 2. Distribución y densidad de las filosomas de $P$. gracilis en el OPC en septiembre de 2007. Esquema general de la topografía dinámica superficial en septiembre (periodo lluvioso mayor). a. Corriente de Colombia. b. Corriente Ciclónica de Panamá. c. Corriente Ecuatorial del Norte. d. Contracorriente Ecuatorial. e. Corriente Ecuatorial del Sur. 
de la superficie en la noche (Baisre, 1976; Báez, 1983; Lipcius y Cobb, 1994; Bruce et al, 2007). No obstante, el reducido número de datos obtenidos en el OPC imposibilita concluir sobre el particular. En cuanto a las fases lunares, cabe destacar que por ejemplo los puérulos de $P$. argus tienen un mayor asentamiento en áreas someras, entre los períodos de luna nueva y cuarto creciente, lo que evidencia su preferencia a desplazarse en horas de menor intensidad lumínica en aguas superficiales (Cruz, 1999).

Por supuesto, en trabajos de esta naturaleza variables fisicoquímicas como, por ejemplo, la temperatura y la salinidad, también deben tenerse en cuenta, pero no se dispone de este tipo de información para la época de estudio. En todo caso, se considera que, por el escaso número de filosomas obtenidas, no es pertinente realizar análisis de esta naturaleza, menos estadísticos.

\section{Características taxonómicas}

Todas las larvas examinadas se caracterizaron por ser transparentes y deprimidas dorso-ventral- mente, particularidades descritas en los diferentes estadios de las filosomas de Palinuridae (Williamson, 1983; Lipcius y Cobb; 1994; Dupré y Guisado, 1996; Gerhard et al, 1999; Rivera y Mujica, 2004). Los ojos tienen pigmentación oscura y a partir del estadio $\mathrm{V}$ se observaron pequeños pigmentos claros dispersos en los pereiópodos y el tórax, características no descritas con anterioridad.

Según Báez (1983), las características de las filosomas de $P$. gracilis a partir del estadio IV son: a. 2-3 setas en el extremo distal de las antenas. $\mathbf{b}$. Una seta en el extremo distal del $3^{\text {er }}$ segmento del $2^{\circ}$ maxilípedo. c. 2-3 setas en el borde externo de la base de las anténulas. d. Una espina sub-exopodal del $1^{\text {er }}$ al $4^{\circ}$ par de pereiópodos. e. Largo cefálico 1.40 veces su ancho. a, b. y c. se evidenciaron en todos los especímenes, aunque no muy claramente en algunos; d se observó a partir del estadio III y e. fue 1.32 a 1.59 veces su ancho (Tabla I; Figura 3). Báez (1983) no describe el estadio III.
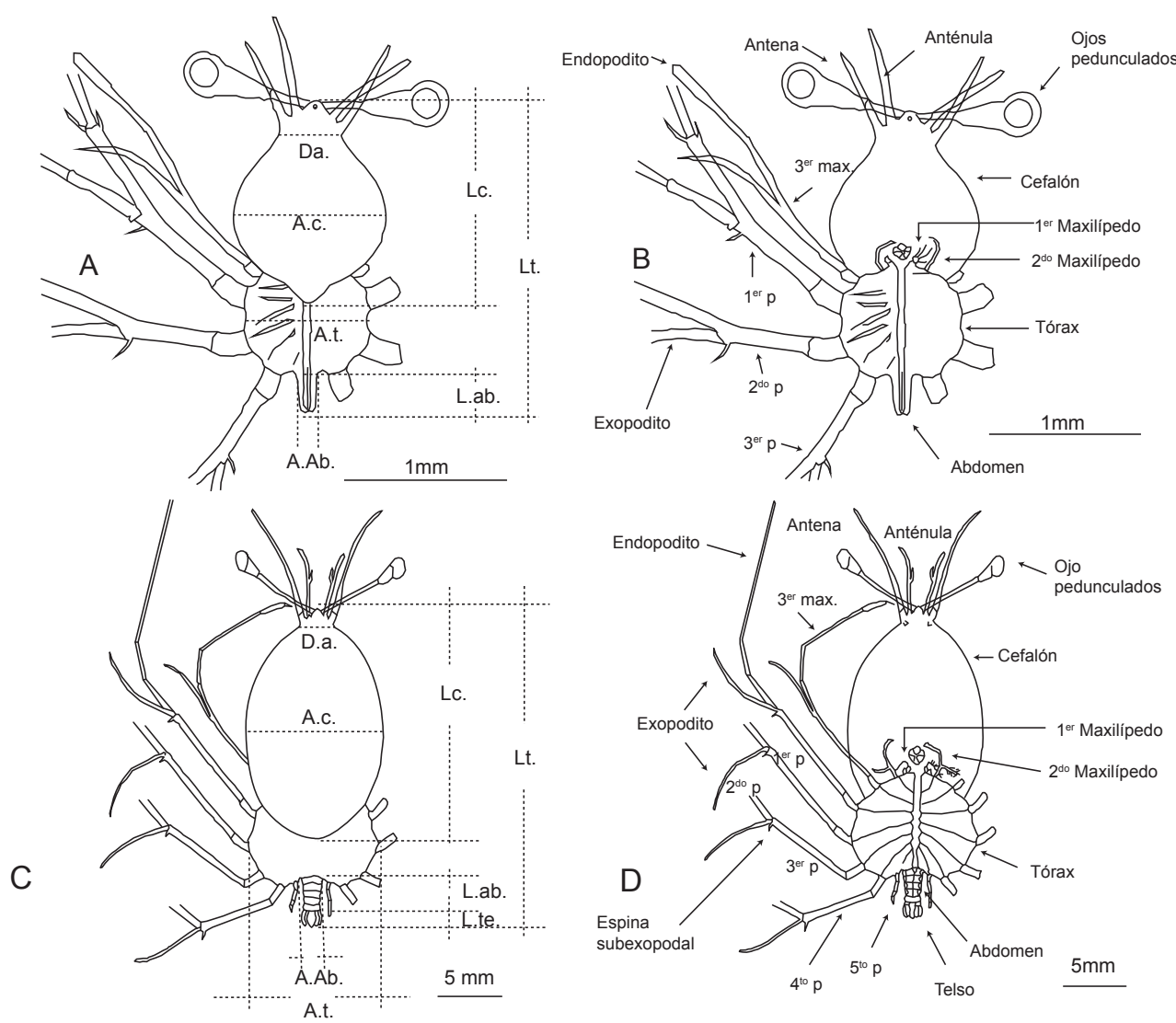

Figura 3. Esquema de una filosoma de $P$. gracilis basado en los organismos capturados en el OPC en septiembre de 2007. Estadios III (A, B) y X (C, D). Lt: Longitud total, Lc: Longitud cefálica, Lab: Longitud abdomen, Lte: Longitud telson, Ac: Ancho cefálico, At: Ancho tórax, Aab: Ancho abdomen, Da: Distancia antenal, P: Pereiópodo. 
Estadio III $(n=2)$ : Disco cefálico periforme, ligeramente agudo y angosto anteriormente con una pequeña prolongación roma posterior (Figura 4A); antenas y anténulas no segmentadas de longitud equivalente; anténulas con dos setas sub-apicales y cuatro terminales (Figura 4B); primer par de maxilípedos con dos segmentos y cuatro setas relativamente largas (Figura $4 C$ ); segundo par de maxilípedos con tres segmentos, seis setas subapicales y sin exopodito; tercer par de maxilípedos con espina coxal; cuatro setas apicales y seis sub- apicales en el endopodito y ocho pares de setas en el exopodito; espinas fuertes ventrales a las coxas de los pereiópodos 1-3; abdomen ligeramente más largo que la coxa del tercer par de pereiópodos, con dos pares de setas terminales (Figura 4D) y en su base rudimentos del cuarto par de pereiópodos; exopodito del tercer par de pereiópodos (Figura $4 \mathrm{E})$ segmentado con cuatro pares de setas, las más basales pequeñas. Ejemplar 1: Lt $2.10 \mathrm{~mm}$, ejemplar 2: Lt $2.49 \mathrm{~mm}$ (Figura $3 \mathrm{~A}-\mathrm{B}$, Tabla II).

Tabla II. Características biométricas de las filosomas de $P$. gracilis capturadas en el OPC en septiembre de 2007. Lt: Longitud total, Lc: Longitud cefálica, Ac: Ancho cefálico, Da: Distancia antenal, At: Ancho del tórax, Aab: Ancho del abdomen, Lab: Longitud del abdomen, Lte: Longitud del telson.

\begin{tabular}{cccccccccc}
\hline Estadio & $\begin{array}{c}\text { Lt } \\
\mathbf{m m}\end{array}$ & $\begin{array}{c}\text { Lc } \\
\mathbf{m m}\end{array}$ & $\begin{array}{c}\text { Ac } \\
\mathbf{m m}\end{array}$ & $\begin{array}{c}\text { Proporción } \\
\text { Lc - Ac }\end{array}$ & $\begin{array}{c}\text { Da } \\
\mathbf{m m}\end{array}$ & $\begin{array}{c}\text { At } \\
\mathbf{m m}\end{array}$ & $\begin{array}{c}\text { Aab } \\
\mathbf{m m}\end{array}$ & $\begin{array}{c}\text { Lab } \\
\mathbf{m m}\end{array}$ & $\begin{array}{c}\text { Lte } \\
\mathbf{m m}\end{array}$ \\
\hline III & 2.10 & 1.54 & 1.05 & 1.47 & 0.40 & 0.92 & 0.14 & 0.25 & No \\
III & 2.49 & 1.67 & 1.20 & 1.39 & 0.55 & 0.99 & 0.14 & 0.30 & No \\
IV & 3.10 & 2.26 & 1.52 & 1.49 & 0.63 & 1.36 & 0.15 & 0.33 & No \\
IV & 3.25 & 2.29 & 1.74 & 1.32 & 0.69 & 1.56 & 0.12 & 0.30 & No \\
V V & 7.70 & 5.80 & 3.64 & 1.59 & 1.12 & 3.90 & 0.38 & 0.70 & No \\
VIII & 11.20 & 8.30 & 5.50 & 1.51 & 1.35 & 5.50 & 0.66 & 1.23 & 0.32 \\
IX & 15.20 & 11.00 & 7.90 & 1.39 & 1.90 & 7.10 & 1.05 & 1.55 & 0.58 \\
IX & 16.00 & 11.10 & 8.30 & 1.34 & 2.00 & 7.80 & 1.20 & 2.00 & 0.40 \\
X & 23.30 & 15.01 & 11.00 & 1.36 & 2.55 & 10.01 & 2.20 & 3.00 & 1.40 \\
\hline
\end{tabular}

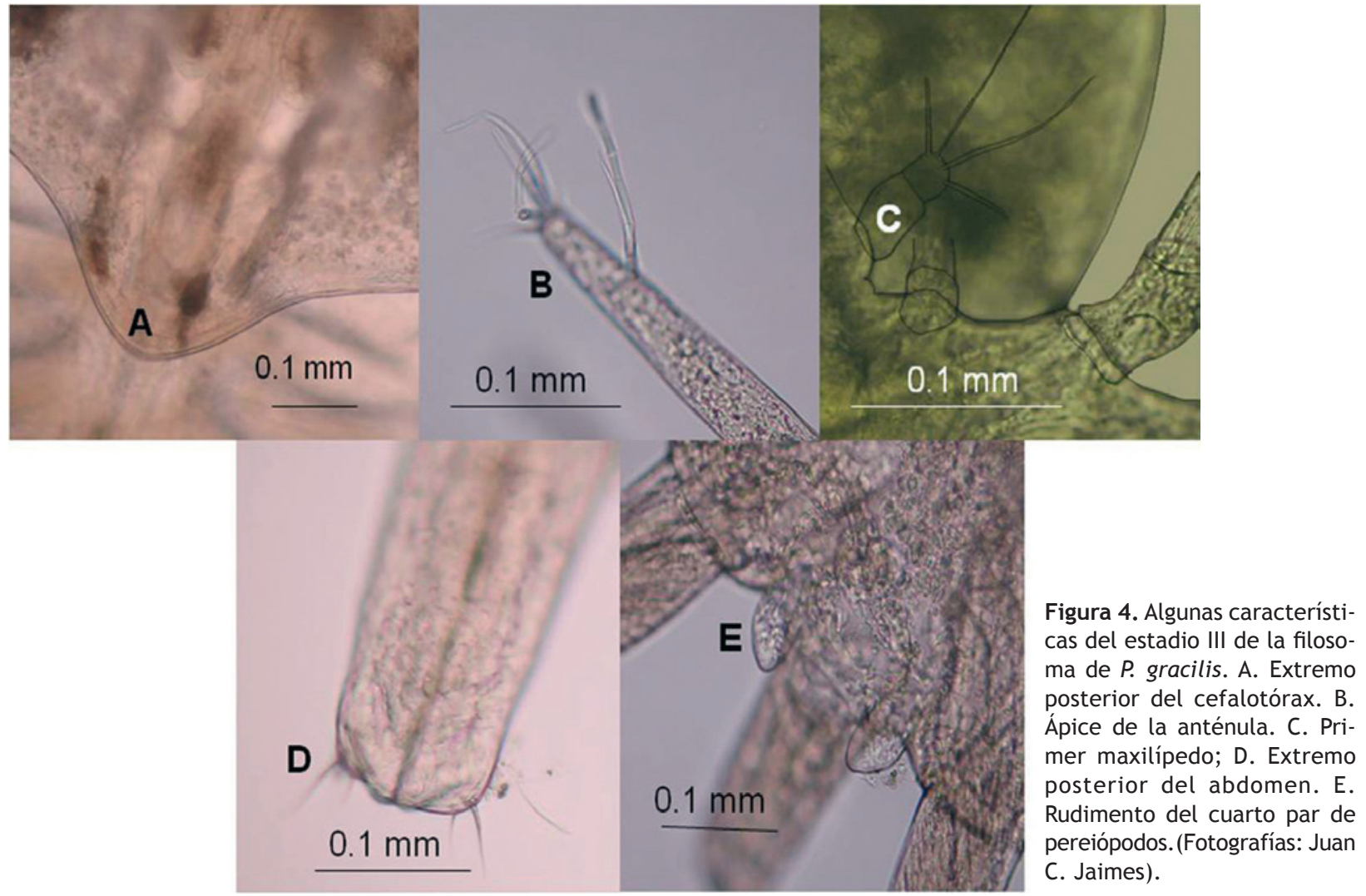


Estadio IV ( $n=2)$ : Persiste la forma del disco cefálico, varían únicamente sus dimensiones con respecto al estadio anterior; antenas y anténulas no segmentadas; anténulas con cuatro setas terminales y una subterminal (Figura 5A); primer par de maxilípedos con dos segmentos y cinco setas, cuatro de las cuales relativamente largas (Figura 5B); segundo par de maxilípedos similar al estadio III; tercer par de maxilipedos con tres setas apicales, seis subapicales (Figura $5 \mathrm{C}$ ) y una espina pequeña en la coxa; espinas robustas ventrales a la coxa y una pequeña en la parte media de los pereiópodos 1 al 3 (Figura 5D); abdomen tan largo como la coxa del tercer par de pereiópodos, con dos pares de setas terminales; cuarto par de pereiópodos bifurcados, no más largos que el abdomen (Figura, 5E), el cual muestra en su base esbozos del quinto par de pereiódopos (Figura 5F). Ejemplar 1: Lt $3.10 \mathrm{~mm}$, ejemplar 2: Lt $3.21 \mathrm{~mm}$ (Tabla II).

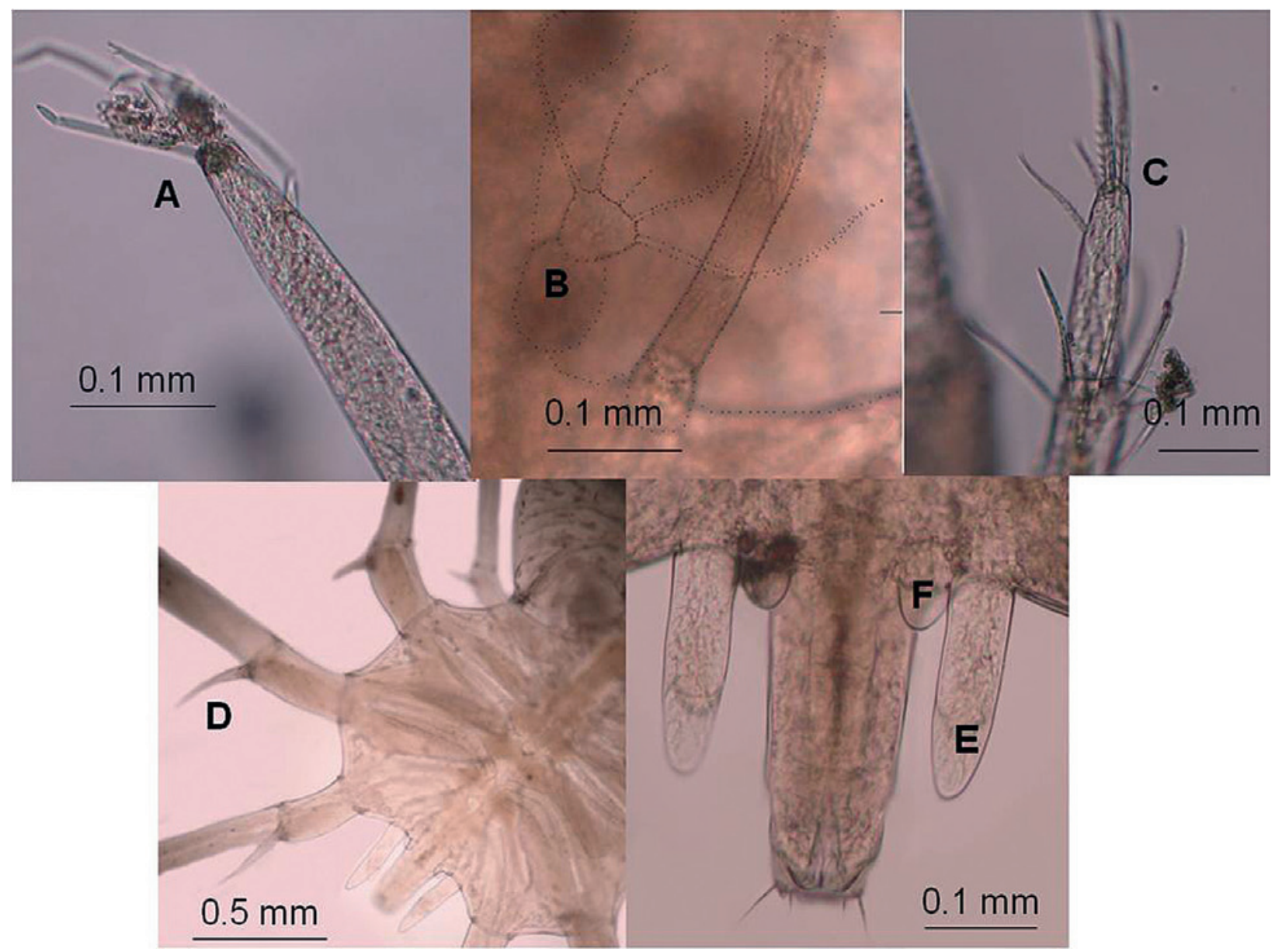

Figura 5. Algunas características del estadio IV de la filosoma de P. gracilis. A. Ápice de la anténula. B. Primer maxilípedo. C. Ápice del tercer maxilípedo. D. Espinas coxales en la base de los pereiópodos. (Fotografías: Juan C. Jaimes).

Estadio V $(n=1)$ : Disco cefálico más robusto y con el extremo posterior ligeramente cóncavo; antenas y anténulas segmentadas, éstas con esbozos de los exopoditos (Figura, 6A); $2^{\circ}$ segmento del primer par de maxilípedos de forma rectangular con once setas distales (Figura 6B); esbozo del exopodito del segundo par de maxilípedos, hacia la mitad del primer basipodito (Figura 6C); abdomen tan largo como la coxa del cuarto par de pereiópodos, exhibiendo dos setas distales pequeñas y un par de urópodos rudimentarios; en su base el abdomen evidencia la formación del quinto par de pereiópodos con muestras de bifurcación y rudimentos de los pleópodos (Figura 6D). Lt $7.70 \mathrm{~mm}$, (Tabla II).

Estadio VIII $(n=1)$ : Disco cefálico robusto, posteriormente más redondeado que en el estadio $\mathrm{V}$; 


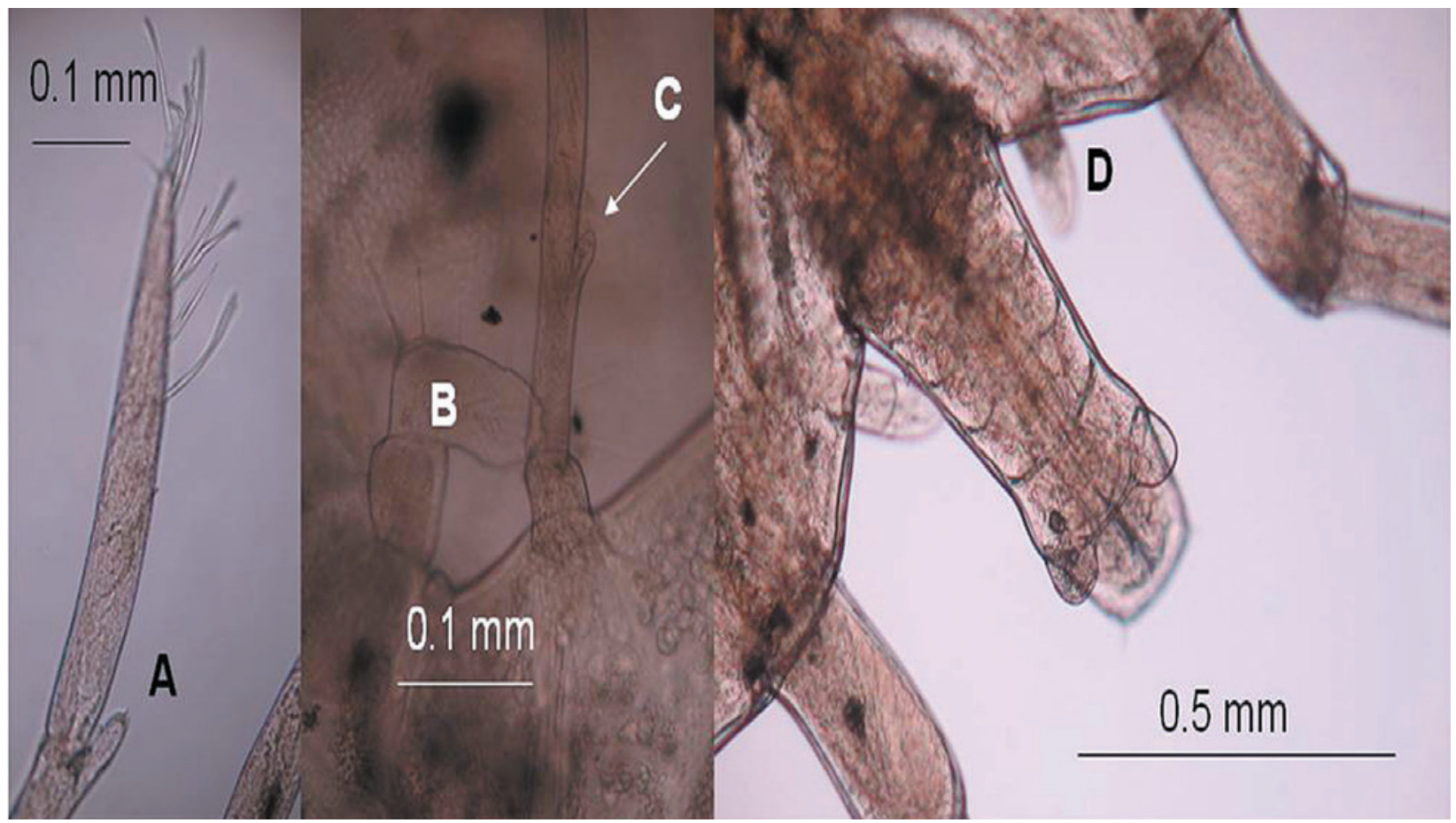

Figura 6. Algunas características del estadio V de la filosoma de P. gracilis. A. Anténula. B. $1^{\text {er }}$ maxilípedo. C. Abdomen. (Fotografías: Juan C. Jaimes).

antenas ligeramente más largas que las anténulas y éstas con endopoditos más desarrollados; el segundo par de maxilípedos conserva la forma del estadio anterior; quinto par de pereiópodos bisegmentados y no superan la longitud del abdomen; primordios de los pleópodos más desarrollados, con pequeñas hendiduras distales y en los urópodos los exopodios más desarrollados que los endopodios, los internos menos desarrollados. Lt $11.20 \mathrm{~mm}$ (Tabla II). En este caso, así como en el estadio siguiente, aunque al microscopio se observaron las estructuras, no se obtuvieron fotografías adecuadas, debido a la opacidad de los ejemplares, producto de la fijación con alcohol al $99.9 \%$.

Estadio IX $(n=2)$ : Disco cefálico elíptico y redondeado en su parte posterior; antenas claramente más largas que las anténulas, cuyos endopoditos están más desarrollados que en estadio anterior; primer par de maxilípedos de forma arriñonada; quinto par de pereiópodos ligeramente más largos que el abdomen que posee tres segmentos; margen externa del urópodo liso. Ejemplar 1: Lt $15.20 \mathrm{~mm}$, ejemplar 2: $16 \mathrm{~mm}$ (Tabla II).

Estadio $X(n=1)$ : Disco cefálico menos angosto en la parte anterior y romo en la posterior (Figura
7E); antenas más largas que las anténulas, todas segmentadas; endopoditos antenulares desarrollados (Figura 7A); primer par de maxilípedos de forma elipsiodal, segundo par de maxilípedos con exopoditos bien desarrollados; quinto par de pereiópodos más largos que el abdomen y con urópodos bien desarrollados, en cuyo margen se observa una pequeña dentición (Figura 7C); pleópodos bifurcados desarrollados (Figura 7B); primeros esbozos de las branquias en exopoditos y pereiópodos (Figura, 7D). Lt $23.30 \mathrm{~mm}$ (Figuras 3 C-D; Tabla II).

Los rangos morfométricos de Lc, Lab, Ac y Aab presentados por Báez (1983) son, en la mayoría de los casos, superiores a los observados en este trabajo, lo cual quizás tenga su respuesta en las condiciones ambientales locales como temperatura, salinidad y oferta trófica que, de acuerdo con el mismo autor, justifica la variación tan alta en los tamaños de un mismo estadio. Según CCCP (2002), históricamente el período septiembre-octubre presenta las temperaturas superficiales más bajas ( 26.5 a $27.0^{\circ} \mathrm{C}$ ), lo que podría estar influenciando, entre otros factores, las tallas observadas. Infortunadamente no se dispone de esta clase de datos para la época de estudio. 

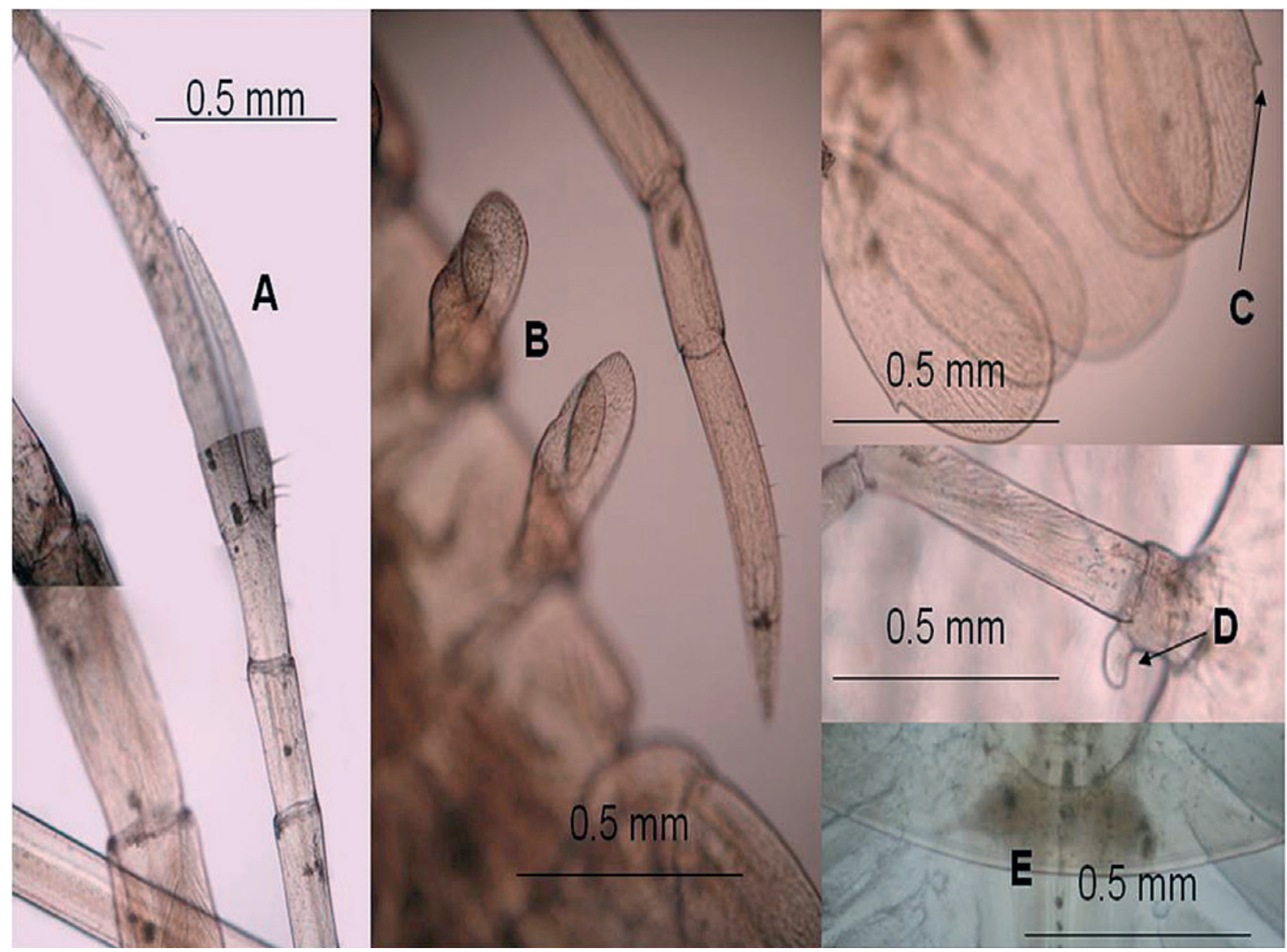

Figura 7. Algunas características del estadio X de la filosoma de P. gracilis. A. Endopodito de la anténula. B. Pleópodos. C. Urópodos. D. Rudimento de las branquias. E. Extremo posterior del cefalotórax. (Fotografías: Juan C. Jaimes).

\section{CONCLUSIONES}

Si bien los datos analizados no permiten una buena interpretación sobre la distribución y abundancia larvaria en el OPC, se advierte para esta época una amplia distribución y mezcla de estadios larvarios de $P$. gracilis.

\section{RECOMENDACIONES}

Es fundamental impulsar la investigación sobre los palinúridos, haciendo énfasis en las primeras fases de desarrollo y en seguimientos periódicos que abarquen más de un año, dada la escasa información, ya que los patrones de reclutamiento son prácticamente desconocidos, en especial en el caso de $P$. gracillis en Colombia.

\section{Agradecimientos}

Este trabajo fue posible gracias a la financiación de la Universidad Militar "Nueva Granada" y al apoyo logístico de la Dirección General Marítima (Dimar), entidad que suministró el buque oceanográfico para la realización del crucero de investigación.

\section{Literatura Citada}

Abele, L. 1975. The macrura decapod crustacea of Malpelo Island. Smithsonian Contribution to Zoology, 176: 69-85.

Alegría, J. 1971. Biología y pesca de la langosta Panulirus argus y Panulirus laevicauda (Ltr.), en el nordeste del Brasil y norte de Colombia. Bogotá D.C. Trabajo de grado (Biología Marina), Universidad Jorge Tadeo Lozano. 
Arango, L., y Márquez, E. 1995. Evaluación de la langosta espinosa Panulirus argus en las Islas de providencia y Santa Catalina, Caribe Colombiano. Revista Cubana de Investigación Pesquera, 19(2): 88-94.

Arango, L., Manrique, M., Jaimes, J. y Cruz, R. 1999. Monitoreo del reclutamiento larval y juvenil de la langosta espinosa (Panulirus. argus) en las islas de Providencia y Santa Catalina, Caribe colombiano. Universidad Jorge Tadeo Lozano. Informe científico técnico. $16 \mathrm{pp}$.

Arango, L., Manrique, M. y Jaimes, J. 2001. Reclutamiento de juveniles de la langosta Panulirus argus con refugios artificiales en el Caribe colombiano. Revista de Investigación Marina, 22(3): 221-228.

Báez, R. 1983. Larvas phyllosomas y puerulus de la langosta verde Panulirus gracilis Streets 1981 procedentes de la expedición Costa Rica, 1973 (Crustacea: Decapoda: Palinuridae). Revista de Biología Marina, Valparaíso, 19 (1): 79-111.

Baisre, J. 1976. Distribución de las larvas de Panulirus argus y Scyllarus americanus (Crustacea; Decapoda) en aguas alrededor de Cuba. Revista de Investigación Certifica, Investigación Pesquera. Cuba, 2(3): 277-297.

Borda, C. y Cruz, R. 2006. Evaluación de la pesquería de langosta (Panulirus argus) en el Archipiélago de San Andrés, Providencia y Santa Catalina, Caribe colombiano. Rev. Invest. Mar. 27 (3): 219-123.

Briones, P., Lozano, E., Martínez-Guerrero A. y Cortés, A.1981. Aspectos generales de la biología y pesca de las langostas en Zihutanejo, Gro, México (Crustacea; Palinuridae). Anales Instituto de Ciencias Del Mar y Limnología. Universidad Nacional Autónoma de México, 8(1): 79-102.

Briones, P. y Lozano, E. 1982. Nuevas localidades en la distribución de Panulirus penicillatus (Oliver, 1791) y $P$. inflatus (Bouvier) (Crustacea: Decapada: Palinuridae) en México. Anales Instituto de Ciencias del mar y Limnologia Universidad Nacional Autónoma de México, 9(1): 389 - 394.

Briones, P. y Gutiérrez, D. 1991. Variaciones en el patrón de reclutamiento de postlarvas de langosta Panulirus argus en Bahía de la Ascensión, México. Revista de Investigaciones Marinas, Cuba 12 (13): 45-56.

Briones, P. y Lozano, E. 1992. Aspect of the reproduction of Panulirus inflatus (Bouvier) and $P$. gracilis streets (Decapoda: Palinuridae) from the Pacific coast of México. Journal of Crustacean Biology, 12 (1): 41-50.

Briones, F. 1994. Variability in postlarval recruitment of the spiny lobster Panulirus argus (Latreille, 1804) to the Mexican Caribbean coast. Crustaceana 66 (3): 326-340.
Briones, P., Lozano, E., Cabrera, M. y Acero, P. 1997. Biología y ecología de las langostas (Crustacea: Decapoda: Palinuridae); En: Flores, D, P. Sánchez, J. Seijo y F. Arreguín (Eds.). Análisis y diagnóstico de los recursos pesqueros críticos del Golfo de México, 81-99 p. EPOMEX Serie Científica, 7 Universidad Autónoma de Campeche.

Bruce, B., Griffin, D. y Bradford, R. 2007. Larval transport and recruitment processes of southern rock lobster. Csiro Marine and Atmospheric Research. Final Report, Australia 76 pp.

Centro Control Contaminación del Pacífico. 2002. Compilación Oceanográfica de la Cuenca Pacífica Colombiana. (Ed.) Dimar. San Andrés de Tumaco: CCCP, Serie Publicaciones Especiales Vol. I, 109 pp.

Córdoba, A. 1997. Aspectos biológicos y ecológicos de individuos juveniles de langosta espinosa (Panulirus argus) en la región de Santa Marta, Caribe colombiano. Bogotá D.C, 76 pp. Trabajo de grado (Biología Marina), Universidad Jorge Tadeo Lozano.

Cruz, R. 1999. Variabilidad del reclutamiento y pronóstico de la pesquería de la langosta (Panulirus argus, Latreille 1804) en Cuba. La Habana, 99 pp. Tesis doctoral (Ciencias Biológicas).

Cruz, R., Baisre, J., Díaz, E., Brito, R., García, C. y Carrodeguas, C. 1990. Atlas Biológico-Pesquero de la Langosta en el Archipiélago Cubano. Publicación Especial. Revista Cubana de Investigación Pesquera, $125 \mathrm{pp}$.

Cruz, R., González, J., de León, M. y Puga, R. 1995. La pesquería de la langosta espinosa (Panulirus argus) en el Gran Caribe. Evaluación y pronóstico. Revista Cubana de Investigación Pesquera, 19(2): 63-76.

Cruz, R., Borda, C., Medina, J., Ayala, A. y Buitrago, D. 2007. Evaluación y ordenación del stock de langosta (Panulirus argus) en el Archipiélago de San Andrés, Providencia y Santa Catalina, Caribe Colombiano, Incoder, Bogotá, 46 pp.

Dimar. 2000. Atlas Cartográfico de los Océanos y Costas Colombianas. Col 1701. Publicación No. 3007, $83 \mathrm{pp}$.

Dupré, E. y Guisado, C. 1996. Identificación de los primeros estados de phyllosoma de la langosta de Juan Fernándaez (Jasus frontalis) mantenidos en laboratorio. Investigaciones Marinas, Valparaíso, 24: 39-50.

Gareth, C. 2000. Abundance and distribution of the Caribbean spiny lobster Panulirus argus in the Aury Channel, Old Providence and Santa Catalina, Colombia. Department of Civil and Offshore Engineering, Edinburgo, 95 pp. 
Gallo, J., Rojas, M. y Correa, F. 2001. Aspectos de las pesquerías y de la biología de la langosta espinosa en la República de Colombia. FAO Fisheries Report No. 619: 184-196.

Gerhard, F., Mantelatto, M., Negriros-Fransozo y Fransozo, A. 1999. Larval Decapoda (Brachyura). En: Boltovskoy, D. (Ed). South Atlantic Zooplancton. Backhuys p. 1280-1351. Publishers, Leiden.

Guzmán, H., Cipriano, R., Vega, A., López, M. y James, M. 2008. Population assessment of the pacific green lobster Panulirus gracilis in the Pacific of Panama. Journal of Shellfish Research, 27(4): 907-915.

Holthuis, L. y Villalobos, A. 1962. Panulirus gracilis Streets y Panulirus inflatus (Bouvier), dos especies de langosta (Crustacea, Decapoda) de la costa del Pacífico de America. Anales del Instituto de Biología de la Universidad de México, 32: 251-276.

Holthuis. 1991. Marine lobsters of the world. FAO species catalogue. Marine lobsters of the world. An annotated and illustrated catalogue of species of interest to fisheries known to date. FAO Fisheries Synopsis No. 125, Vol. 13. Roma, 292 pp.

ICA. 2006. Estadísticas de pesca y acuicultura, Tabla I. Desembarque de la pesca industrial y artesanal océano Pacífico colombiano 1995-2006; Tabla II. Desembarque de la pesca industrial y artesanal océano Atlántico colombiano 1995-2006. http: / / www.ica.gov.co/getdoc/9b2d924c-8263-489291b1-fcc49d6393cd/Oceano Pacífico.aspx

Inpa. 2001. Boletín estadístico pesquero colombiano. Instituto Nacional de Pesca y Acuicultura, Santafé de Bogotá, 139 pp.

Invemar. 2005. Informe del Estado de los Ambientes Marinos y Costeros en Colombia. Serie de Publicaciones Periódicas, Instituto de Investigaciones Marinas y Costeras "Benito vides de Andreis" , Santa Marta, 8: 360.

Jaimes, J. 2000. Dinámica del puérulos de langosta espinosa Panulirus argus (Latreille, 1804) dentro de la plataforma insular de Providencia y Santa Catalina, Caribe colombiano. Bogotá D.C, 82 pp. Trabajo de grado (Biología Marina), Universidad Jorge Tadeo Lozano.

Jaimes J., Pinzón, Y. y Trujillo, C. 2004. Explorando alternativas de pesca responsable con la langosta espinosa en Santa Marta. Fundación Sila Kangama: Santa Marta, Colombia. 28 pp.

Lazarus, J. y Cantera, J. 2007. Crustáceos (Crustacea: Sessilia, Stomatopoda, Isopoda, Amphipoda, Decapoda) de Bahía Málaga, Valle del Cauca (Pacífico colombiano). Biota Colombiana 8 (2): 221-190.

Lemaitre, R. y Álvarez, R. 1992. Crustáceos decápodos del Pacífico colombiano: lista de especies y con- sideraciones zoogeográficas. Anales Instituto de Investigaciones Marinas “Benito Vives de Andréis", 21: 33-76.

Lipcius, R., y Cobb, J. 1994. Ecology and fishery biology of spiny lobster. En: Phillips, B, J. Cobb y J. Kittaka (Eds.). Spiny lobster management. Fishing News. Book, Blackwell, Oxford. 1-24 pp.

López E. y Barragán, J. 1974. Estudio biológico pesquero de la langosta de espina del Pacífico ecuatoriano (Crustacea: Decapoda: Panuliridae). Revista del Pacífico Sur, Chile, 2: 63-79.

Lozano, E., Briones, P. Santareli, L. y Gargia, A. 1982. Densidad poblacional de Panulirus gracilis y $P$. inflatus (Bouvier) (Crustacea: palinuridae) en dos áreas cercanas a Zihuatenjo, Gro, México. Ciencias Pesqueras, Instituto Nacional de la Pesca Secretaría de Pesca (México), 3: 61-73.

Martínez, D. 1970. Contribución al conocimiento y estudio del zooplancton del Caribe colombiano. Trabajo de grado (Biología Marina). Bogotá D.C, 75 pp. Universidad Jorge Tadeo Lozano.

Martínez, A. y Chávez, H. 1985. Nota sobre la colecta de larvas y juveniles de langosta roja Panulirus interruptus (Randall) en la costa occidental de Baja California, México. Ciencias Marinas, 11 (2): 93-100.

Medellín, D. 2005. Composición y distribución de larvas de crustáceos decápodos en estado zoea en el área nororiental el Mar Caribe colombiano. Trabajo de grado (Biología Marina). Bogotá D.C, 77 pp. Universidad Jorge Tadeo Lozano.

Milano, E., y Bejarano, J. 1994. Pacífico colombiano condiciones termohalinas y estandarización de la curva S-T. Boletín Científico Cioh, (15): 39-66.

Muñoz, I., Pérez, R., Flores, L. y Borrego, I. 2000. Distribución y abundancia de filosomas de Panulirus (Decapoda: Palinuridae) en el sureste del Golfo de California, México. Rev. Biología. Tropical. Marina, 48 (159-167).

Olvera, L. y Ordóñez, L. 1988. Distribución, abundancia relativa y desarrollo larvario de langostas Panulirus argus y Scyllarus americanus en la ZEE del Golfo de México y mar Caribe. Ciencia pesquera Instituto Nalcional de la Pesca. Secretaría de Pesca (6): 7-31.

Rivera, J. y Mujica, A. 2004. Larvas Phyllosomas (Decapoda, Palinuridae, Scyllaridae) de las islas oceánica chilenas. Investigaciones Marinas, Valparaíso, 32 (2): 99-111.

Riveros, G. 1972. Ganancia hipotética en los pesos de Panulirus argus durante las mudas frente a la Guajira. Boletín Informativo, Bogotá, 2(3): 29- 45.

Scheltema, R. 1986. "On dispersal and planktonic larvae of benthic invertebrates: an eclectic overview 
and summary of problems". Bulletin of Marine Sciences, 39: 290-322.

Steedman, H. (Ed.). 1976. Zooplankton fixation and preservation. Monographies in Oceanography Methodology, 4. Unesco PresRevistas, París, 350 pp.

Smith, P. y Richardson, S. 1979. Técnicas modelo para prospecciones de huevos y larvas de peces pelágicos. FAO, Documentos Técnicos de Pesca, 175: 1-107.

Velásquez, L. y Gutiérrez, R. 2006. Talla y peso de primera madurez sexual al 50 \% (Lm50 \%) y (Pm50\%) para hembras de langosta verde Panulirus gracilis del Pacífico nicaragüense. Ministerio de Fomento, Industria y Comercio (MiFIC), Administración Nacional de Pesca y Acuicultura (AdPesca), Centro de Investigaciones Pesqueras y Acuicolas Cipa/ AdPesca. Managua, Nicaragua. P. 8.

Williamson, D. 1983. Decapoda, Larvae, VIII. Conseil international pour l' exploration de la Mer, Fiches d'Identification du Zooplancton, Copenhague, 167/168: P. 8. 